DESY 12-091

arXiv:1205.7051 [math.NT]

\title{
On Multiple Zeta Values of Even Arguments
}

\author{
Michael E. Hoffman ${ }^{1}$ \\ Dept. of Mathematics, U. S. Naval Academy \\ Annapolis, MD 21402 USA \\ and \\ Deutsches Elektronen-Synchrotron DESY \\ Platanenalle 6, D-15738 Zeuthen, Germany \\ meh@usna.edu
}

March 17, 2016

Keywords: multiple zeta values, symmetric functions, Bernoulli numbers

MR Classifications: Primary 11M32; Secondary 05E05, 11B68

\begin{abstract}
For $k \leq n$, let $E(2 n, k)$ be the sum of all multiple zeta values with even arguments whose weight is $2 n$ and whose depth is $k$. Of course $E(2 n, 1)$ is the value $\zeta(2 n)$ of the Riemann zeta function at $2 n$, and it is well known that $E(2 n, 2)=\frac{3}{4} \zeta(2 n)$. Recently Z. Shen and T. Cai gave formulas for $E(2 n, 3)$ and $E(2 n, 4)$ in terms of $\zeta(2 n)$ and $\zeta(2) \zeta(2 n-2)$. We give two formulas for $E(2 n, k)$, both valid for arbitrary $k \leq n$, one of which generalizes the Shen-Cai results; by comparing the two we obtain a Bernoulli-number identity. We also give explicit generating functions for the numbers $E(2 n, k)$ and for the analogous numbers $E^{\star}(2 n, k)$ defined using multiple zeta-star values of even arguments.
\end{abstract}

\footnotetext{
${ }^{1}$ Supported by a grant from the German Academic Exchange Service (DAAD) during the preparation of this paper. The author also thanks DESY for providing facilities and financial support for travel, and the referee for urging him to include multiple zeta-star values (which led to Corollary 1).
} 


\section{Introduction and Statement of Results}

For positive integers $i_{1}, \ldots, i_{k}$ with $i_{1}>1$, we define the multiple zeta value $\zeta\left(i_{1}, \ldots, i_{k}\right)$ by

$$
\zeta\left(i_{1}, \ldots, i_{k}\right)=\sum_{n_{1}>\cdots>n_{k} \geq 1} \frac{1}{n_{1}^{i_{1}} \cdots n_{k}^{i_{k}}} .
$$

The multiple zeta value (1) is said to have weight $i_{1}+\cdots+i_{k}$ and depth $k$. Many remarkable identities have been proved about these numbers, but in this note we will concentrate on the case where the $i_{j}$ are even integers. Let $E(2 n, k)$ be the sum of all the multiple zeta values of even-integer arguments having weight $2 n$ and depth $k$, i.e.,

$$
E(2 n, k)=\sum_{\substack{i_{1}, \ldots, i_{k} \text { even } \\ i_{1}+\cdots+i_{k}=2 n}} \zeta\left(i_{1}, \ldots, i_{k}\right) .
$$

Of course

$$
E(2 n, 1)=\zeta(2 n)=\frac{(-1)^{n-1} B_{2 n}(2 \pi)^{2 n}}{2(2 n) !},
$$

where $B_{2 n}$ is the $2 n$th Bernoulli number, by the classical formula of Euler. Euler also studied double zeta values (i.e., multiple zeta values of depth 2) and in his paper [3] gave two identities which read

$$
\begin{aligned}
\sum_{i=2}^{2 n-1}(-1)^{i} \zeta(i, 2 n-i) & =\frac{1}{2} \zeta(2 n) \\
\sum_{i=2}^{2 n-1} \zeta(i, 2 n-i) & =\zeta(2 n)
\end{aligned}
$$

in modern notation. From these it follows that

$$
E(2 n, 2)=\frac{3}{4} \zeta(2 n)
$$

though Gangl, Kaneko and Zagier [4] seem to be the first to have pointed it out in print. Recently Shen and Cai [11] proved the formulas

$$
\begin{aligned}
& E(2 n, 3)=\frac{5}{8} \zeta(2 n)-\frac{1}{4} \zeta(2) \zeta(2 n-2), n \geq 3 \\
& E(2 n, 4)=\frac{35}{64} \zeta(2 n)-\frac{5}{16} \zeta(2) \zeta(2 n-2), n \geq 4
\end{aligned}
$$


Identity (3) was also proved by Machide [10] using a different method.

This begs the question whether there is a general formula of this type for $E(2 n, k)$. The pattern

$$
\frac{3}{4}, \quad \frac{3}{4} \cdot \frac{5}{6}=\frac{5}{8}, \quad \frac{3}{4} \cdot \frac{5}{6} \cdot \frac{7}{8}=\frac{35}{64}
$$

of the leading coefficients makes one curious. In fact, the general result is as follows.

Theorem 1. For $k \leq n$,

$$
\begin{aligned}
E(2 n, k)=\frac{1}{2^{2(k-1)}} & \left(\begin{array}{c}
2 k-1 \\
k
\end{array}\right) \zeta(2 n) \\
& \quad-\sum_{j=1}^{\left\lfloor\frac{k-1}{2}\right\rfloor} \frac{1}{2^{2 k-3}(2 j+1) B_{2 j}}\left(\begin{array}{c}
2 k-2 j-1 \\
k
\end{array}\right) \zeta(2 j) \zeta(2 n-2 j) .
\end{aligned}
$$

The next two cases after (4) are

$$
\begin{aligned}
& E(2 n, 5)=\frac{63}{128} \zeta(2 n)-\frac{21}{64} \zeta(2) \zeta(2 n-2)+\frac{3}{64} \zeta(4) \zeta(2 n-4) \\
& E(2 n, 6)=\frac{231}{512} \zeta(2 n)-\frac{21}{64} \zeta(2) \zeta(2 n-2)+\frac{21}{256} \zeta(4) \zeta(2 n-4) .
\end{aligned}
$$

We prove Theorem 1 in $\S 3$ below, using the generating function

$$
F(t, s)=1+\sum_{n \geq k \geq 1} E(2 n, k) t^{n} s^{k}
$$

In $\S 2$ we establish the following explicit formula.

\section{Theorem 2.}

$$
F(t, s)=\frac{\sin (\pi \sqrt{1-s} \sqrt{t})}{\sqrt{1-s} \sin (\pi \sqrt{t})} .
$$

Our proof uses symmetric functions. We define a homomorphism $\mathfrak{Z}$ : Sym $\rightarrow \mathbf{R}$, where Sym is the algebra of symmetric functions, and a family $N_{n, k} \in$ Sym such that $\mathfrak{Z}$ sends $N_{n, k}$ to $E(2 n, k)$. We then obtain a formula for the generating functions

$$
\mathcal{F}(t, s)=1+\sum_{n \geq k \geq 1} N_{n, k} t^{n} s^{k} \in \operatorname{Sym}[[t, s]]
$$


and apply $\mathfrak{Z}$ to get Theorem 2 .

Along with the multiple zeta values there are the multiple zeta-star values

$$
\zeta^{\star}\left(i_{1}, \ldots, i_{k}\right)=\sum_{n_{1} \geq \cdots \geq n_{k} \geq 1} \frac{1}{n_{1}^{i_{1}} \cdots n_{k}^{i_{k}}},
$$

where the strict inequalities in equation (1) are replaced by $\geq$. These coincide with multiple zeta values for depth 1 , and for greater depths they are simply sums of multiple zeta values, e.g.,

$$
\zeta^{\star}(6,2,4)=\zeta(6,2,4)+\zeta(8,4)+\zeta(6,6)+\zeta(12) .
$$

Conversely, multiple zeta values are sums of multiple zeta-star values with signs alternating by depth, e.g.,

$$
\zeta(6,2,4)=\zeta^{\star}(6,2,4)-\zeta^{\star}(8,4)-\zeta^{\star}(6,6)+\zeta^{\star}(12) .
$$

For $k \leq n$ we can define $E^{\star}(2 n, k)$ as the sum of all multiple zeta-star values with even arguments having weight $2 n$ and depth $k$. The generating function

$$
F^{\star}(t, s)=1+\sum_{n \geq k \geq 1} E^{\star}(2 n, k) t^{n} s^{k}
$$

turns out to have a remarkably simple relation to $F(t, s)$, as we show in $\S 2$.

Corollary 1. The generating functions $F(t, s)$ and $F^{\star}(t, s)$ are related by $F^{\star}(t, s)=F(t,-s)^{-1}$.

From the form of $\mathcal{F}(t, s)$ we show that it satisfies a partial differential equation (Proposition 1 below), which is equivalent to a recurrence for the $N_{n, k}$. From the latter we obtain a formula for the $N_{n, k}$ in terms of complete and elementary symmetric functions, to which $\mathfrak{Z}$ can be applied to give the following alternative formula for $E(2 n, k)$.

Theorem 3. For $k \leq n$,

$$
E(2 n, k)=\frac{(-1)^{n-k-1} \pi^{2 n}}{(2 n+1) !} \sum_{i=0}^{n-k}\left(\begin{array}{c}
n-i \\
k
\end{array}\right)\left(\begin{array}{c}
2 n+1 \\
2 i
\end{array}\right) 2\left(2^{2 i-1}-1\right) B_{2 i} .
$$


Note that the sum given by Theorem 3 has $n-k+1$ terms, while that given by Theorem 1 has $\left\lfloor\frac{k-1}{2}\right\rfloor+1$ terms. Yet another explicit formula for $E(2 n, k)$ can be obtained by setting $d=1$ in Theorem 7.1 of Komori, Matsumoto and Tsumura [8]. That formula expresses $E(2 n, k)$ as a sum over partitions of $k$, and it is not immediately clear how it relates to our two formulas.

Comparison of Theorems 1 and 3 establishes the following Bernoullinumber identity.

Theorem 4. For $k \leq n$,

$$
\begin{aligned}
& \sum_{i=0}^{\left\lfloor\frac{k-1}{2}\right\rfloor}\left(\begin{array}{c}
2 k-2 i-1 \\
k
\end{array}\right)\left(\begin{array}{c}
2 n+1 \\
2 i+1
\end{array}\right) B_{2 n-2 i}= \\
& (-1)^{k} 2^{2 k-2 n} \sum_{i=0}^{n-k}\left(\begin{array}{c}
n-i \\
k
\end{array}\right)\left(\begin{array}{c}
2 n+1 \\
2 i
\end{array}\right)\left(2^{2 i-1}-1\right) B_{2 i}
\end{aligned}
$$

It is interesting to contrast this result with the Gessel-Viennot identity (see [2, Theorem 4.2]) valid on the complementary range:

$$
\sum_{i=0}^{\left\lfloor\frac{k-1}{2}\right\rfloor}\left(\begin{array}{c}
2 k-2 i-1 \\
k
\end{array}\right)\left(\begin{array}{c}
2 n+1 \\
2 i+1
\end{array}\right) B_{2 n-2 i}=\frac{2 n+1}{2}\left(\begin{array}{c}
2 k-2 n \\
k
\end{array}\right), \quad k>n .
$$

Note that the right-hand side of equation (5) is zero unless $k \geq 2 n$.

\section{Symmetric Functions}

We think of Sym as the subring of $\mathbf{Q}\left[\left[x_{1}, x_{2}, \ldots\right]\right]$ consisting of those formal power series of bounded degree that are invariant under permutations of the $x_{i}$. A useful reference is the first chapter of Macdonald [9]. We denote the elementary, complete, and power-sum symmetric functions of degree $i$ by $e_{i}$, 
$h_{i}$, and $p_{i}$ respectively. They have associated generating functions

$$
\begin{aligned}
& E(t)=\sum_{j=0}^{\infty} e_{j} t^{j}=\prod_{i=1}^{\infty}\left(1+t x_{i}\right) \\
& H(t)=\sum_{j=0}^{\infty} h_{j} t^{j}=\prod_{i=1}^{\infty} \frac{1}{1-t x_{i}}=E(-t)^{-1} \\
& P(t)=\sum_{j=1}^{\infty} p_{j} t^{j-1}=\sum_{i=1}^{\infty} \frac{x_{i}}{1-t x_{i}}=\frac{H^{\prime}(t)}{H(t)} .
\end{aligned}
$$

As explained in [6] and in greater detail in [7], there is a homomorphism $\zeta$ : $\mathrm{Sym}^{0} \rightarrow \mathbf{R}$, where $\mathrm{Sym}^{0}$ is the subalgebra of Sym generated by $p_{2}, p_{3}, p_{4}, \ldots$, such that $\zeta\left(p_{i}\right)$ is the value $\zeta(i)$ of the Riemann zeta function at $i$, for $i \geq 2$ (in $[6,7]$ this homomorphism is extended to all of Sym, but we do not need the extension here). Let $\mathcal{D}: \mathrm{Sym} \rightarrow$ Sym be the degree-doubling map that sends $x_{i}$ to $x_{i}^{2}$. Then $\mathcal{D}(\mathrm{Sym}) \subset \operatorname{Sym}^{0}$, so the composition $\mathfrak{Z}=\zeta \mathcal{D}$ is defined on all of Sym. (Alternatively, we can simply think of $\mathfrak{Z}$ as sending $x_{i}$ to $1 / i^{2}$ : see [9, Ch. I, $\S 2$, ex. 21].) Note that $\mathfrak{Z}\left(p_{i}\right)=\zeta(2 i) \in \mathbf{R}$. Further, $\mathfrak{Z}$ sends the monomial symmetric function $m_{i_{1}, \ldots, i_{k}}$ to the symmetrized sum of multiple zeta values

$$
\frac{1}{\left|\operatorname{Iso}\left(i_{1}, \ldots, i_{k}\right)\right|} \sum_{\sigma \in S_{k}} \zeta\left(2 i_{\sigma(1)}, 2 i_{\sigma(2)}, \ldots, 2 i_{\sigma(k)}\right),
$$

where $S_{k}$ is the symmetric group on $k$ letters and $\operatorname{Iso}\left(i_{1}, \ldots, i_{k}\right)$ is the subgroup of $S_{k}$ that fixes $\left(i_{1}, \ldots, i_{k}\right)$ under the obvious action.

Now let $N_{n, k}$ be the sum of all the monomial symmetric functions corresponding to partitions of $n$ having length $k$. Of course $N_{n, k}=0$ unless $k \leq n$, and $N_{k, k}=e_{k}$. Then $\mathfrak{Z}$ sends $N_{n, k}$ to $E(2 n, k)$. Also, if we define (as in the introduction)

$$
\mathcal{F}(t, s)=1+\sum_{n \geq k \geq 1} N_{n, k} t^{n} s^{k},
$$

then $\mathfrak{Z}$ sends $\mathcal{F}(t, s)$ to the generating function $F(t, s)$. We have the following simple description of $\mathcal{F}(t, s)$.

Lemma 1. $\mathcal{F}(t, s)=E((s-1) t) H(t)$. 
Proof. Evidently $\mathcal{F}(t, s)$ has the formal factorization

$$
\prod_{i=1}^{\infty}\left(1+s t x_{i}+s t^{2} x_{i}^{2}+\cdots\right)=\prod_{i=1}^{\infty} \frac{1+(s-1) t x_{i}}{1-t x_{i}}=E((s-1) t) H(t) .
$$

Proof of Theorem 2. Using the well-known formula for $\zeta(2,2, \ldots, 2)[5$, Cor. 2.3],

$$
\mathfrak{Z}\left(e_{n}\right)=\zeta(\underbrace{2,2, \ldots, 2}_{n})=\frac{\pi^{2 n}}{(2 n+1) !} .
$$

Hence

$$
\mathfrak{Z}(E(t))=\frac{\sinh (\pi \sqrt{t})}{\pi \sqrt{t}},
$$

and the image of $H(t)=E(-t)^{-1}$ is

$$
\mathfrak{Z}(H(t))=\frac{\pi \sqrt{-t}}{\sinh (\pi \sqrt{-t})}=\frac{\pi \sqrt{t}}{\sin (\pi \sqrt{t})} .
$$

Thus from Lemma $1 F(t, s)=\mathfrak{Z}(\mathcal{F}(t, s))$ is

$$
\mathfrak{Z}(E((s-1) t) H(t))=\frac{\sinh (\pi \sqrt{(s-1) t})}{\pi \sqrt{(s-1) t}} \frac{\pi \sqrt{t}}{\sin (\pi \sqrt{t})}=\frac{\sin (\pi \sqrt{(1-s) t})}{\sqrt{1-s} \sin (\pi \sqrt{t})} .
$$

Taking limits as $s \rightarrow 1$ in Theorem 2 , we obtain

$$
F(t, 1)=\frac{\pi \sqrt{t}}{\sin \pi \sqrt{t}}
$$

and so, taking the coefficient of $t^{n}$, the following result.

Corollary 2. For all $n \geq 1$,

$$
\sum_{k=1}^{n} E(2 n, k)=\frac{2\left(2^{2 n-1}-1\right)(-1)^{n-1} B_{2 n} \pi^{2 n}}{(2 n) !}=2\left(1-2^{1-2 n}\right) \zeta(2 n) .
$$


Remark. This result was obtained previously by Aoki, Kombu and Ohno [1], who stated it in the language of multiple zeta-star values; since

$$
\zeta^{\star}(\underbrace{2, \ldots, 2}_{n})=E(2 n, n)+E(2 n, n-1)+\cdots+E(2 n, 1),
$$

Corollary 2 can be recognized as [1, equation (4.6)]. In fact, using Euler's infinite product for sine, one sees that

$$
F\left(z^{2}, 1\right)=\prod_{m=1}^{\infty}\left(1-\frac{z^{2}}{m^{2}}\right)^{-1}=1+\sum_{n \geq 1} \zeta^{\star}(\underbrace{2, \ldots, 2}_{n}) z^{2 n}
$$

Recall that we defined $E^{\star}(2 n, k)$ as the sum of all multiple zeta-star values with even arguments having depth $k$ and weight $2 n$. The left-hand side of equation $(7)$ is $E^{\star}(2 n, n)$, and that equation generalizes as follows.

Lemma 2. For $n \geq k \geq 1$,

$$
E^{\star}(2 n, k)=\sum_{j=1}^{k}\left(\begin{array}{l}
n-j \\
k-j
\end{array}\right) E(2 n, j) .
$$

Proof. Let $I=\left(i_{1}, \ldots, i_{k}\right)$ be a composition (i.e., ordered partition) of $n$. We can think of $I$ as specified by placing $k-1$ dividers within a row of $n$ dots, which makes it clear that there are $\left(\begin{array}{l}n-1 \\ k-1\end{array}\right)$ compositions of $n$ with $k$ parts. If we associate to $I$ the multiple zeta and multiple star zeta values

$$
\zeta(2 I)=\zeta\left(2 i_{1}, \ldots, 2 i_{k}\right), \quad \zeta^{\star}(2 I)=\zeta^{\star}\left(2 i_{1}, \ldots, 2 i_{k}\right)
$$

of even values, then $\zeta^{\star}(2 I)=\sum_{I \succeq J} \zeta(2 J)$, where $\succeq$ is the partial order on compositions given by refinement, i.e., $I \succeq J$ if $J$ can be obtained by combining adjacent parts of $I$; in terms of the dividers-in-row-of-dots picture, $J$ is obtained by removing some dividers from $I$.

Now $E^{\star}(2 n, k)$ is the sum of all $\zeta^{\star}(2 I)$ with $I$ having $k$ parts. Write each of these as a sum of multiple zeta values. Then the coefficient of $\zeta(2 J)$, where $J$ has $j \leq k$ parts, is the number of distinct compositions $I$ with $k$ parts such that $J \preceq I$; this corresponds to the number of ways to insert $k-j$ additional dividers into $J$. Since there are $n-1-(j-1)=n-j$ places to put them, this number is $\left(\begin{array}{l}n-j \\ k-j\end{array}\right)$. 
Proof of Corollary 1. Using Lemma 2 and Theorem 2,

$$
\begin{gathered}
F^{\star}(t, s)=1+\sum_{n \geq k \geq 1} E^{\star}(2 n, k) t^{n} s^{k}=1+\sum_{n \geq k \geq 1} \sum_{j=1}^{k}\left(\begin{array}{l}
n-j \\
k-j
\end{array}\right) E(2 n, j) t^{n} s^{k} \\
=1+\sum_{n \geq j \geq 1} E(2 n, j) \sum_{i=0}^{n-j}\left(\begin{array}{c}
n-j \\
i
\end{array}\right) t^{n} s^{j+i}=1+\sum_{n \geq j \geq 1} E(2 n, j) t^{n} s^{j}(1+s)^{n-j} \\
=F\left(t(1+s), \frac{s}{1+s}\right)=\frac{\sin \left(\pi \sqrt{1-\frac{s}{1+s}} \sqrt{t(1+s)}\right)}{\sqrt{1-\frac{s}{1+s}} \sin (\pi \sqrt{t(1+s)})}=\frac{\sqrt{1+s} \sin (\pi \sqrt{t})}{\sin (\pi \sqrt{(1+s) t})} \\
=\frac{1}{F(t,-s)}
\end{gathered}
$$

Another consequence of Lemma 1 is the following partial differential equation.

\section{Proposition 1.}

$$
t \frac{\partial \mathcal{F}}{\partial t}(t, s)+(1-s) \frac{\partial \mathcal{F}}{\partial s}(t, s)=t P(t) \mathcal{F}(t, s) .
$$

Proof. From Lemma 1 we have

$$
\begin{aligned}
& \frac{\partial \mathcal{F}}{\partial t}(t, s)=(s-1) E^{\prime}((s-1) t) H(t)+E((s-1) t) H^{\prime}(t) \\
& \frac{\partial \mathcal{F}}{\partial s}(t, s)=t E^{\prime}((s-1) t) H(t)
\end{aligned}
$$

from which the conclusion follows.

Now examine the coefficient of $t^{n} s^{k}$ in Proposition 1 to get the following.

Proposition 2. For $n \geq k+1$,

$$
p_{1} N_{n-1, k}+p_{2} N_{n-2, k}+\cdots+p_{n-k} N_{k, k}=(n-k) N_{n, k}+(k+1) N_{n, k+1} .
$$

It is also possible to prove this result directly via a counting argument like that used to prove the lemma of $[7, \mathrm{p} .16]$.

The preceding result allows us to write $N_{n, k}$ explicitly in terms of complete and elementary symmetric functions as follows. 
Lemma 3. For $r \geq 0$,

$$
N_{k+r, k}=\sum_{i=0}^{r}(-1)^{i}\left(\begin{array}{c}
k+i \\
i
\end{array}\right) h_{r-i} e_{k+i} .
$$

Proof. We use induction on $r$, the result being evident for $r=0$. Proposition 2 gives

$$
\sum_{i=1}^{r+1} p_{i} N_{k+r+1-i, k}=(r+1) N_{k+r+1, k}+(k+1) N_{k+r+1, k+1}
$$

which after application of the induction hypothesis becomes

$$
\begin{aligned}
& \sum_{i=1}^{r+1} \sum_{j=0}^{r+1-j}(-1)^{j} p_{i}\left(\begin{array}{c}
k+j \\
j
\end{array}\right) h_{r+1-i-j} N_{k+j, k+j}= \\
&(r+1) N_{k+r+1, k}+(k+1) \sum_{j=0}^{r}\left(\begin{array}{c}
k+1+j \\
j
\end{array}\right) h_{r-j} N_{k+1+j, k+1+j} .
\end{aligned}
$$

The latter equation can be rewritten

$$
\begin{aligned}
\sum_{j=0}^{r}(-1)^{j}\left(\begin{array}{c}
k+j \\
j
\end{array}\right) N_{k+j, k+j} \sum_{i=1}^{r+1-j} p_{i} h_{r+1-i-j}= & \\
& (r+1) N_{k+r+1, k}-(k+1) \sum_{j=1}^{r+1}(-1)^{j}\left(\begin{array}{c}
k+j \\
j-1
\end{array}\right) h_{r+1-j} N_{k+j, k+j} .
\end{aligned}
$$

Now the inner sum on the left-hand side is $(r+1-j) h_{r+1-j}$ by the recurrence relating the complete and power-sum symmetric functions, so we have

$$
\begin{aligned}
& (r+1) N_{k+r+1, k}-(r+1) N_{k, k} h_{r+1}= \\
& \quad \sum_{j=1}^{r+1}(-1)^{j} h_{r+1-j} N_{k+j, k+j}\left((r+1-j)\left(\begin{array}{c}
k+j \\
j
\end{array}\right)+(k+1)\left(\begin{array}{c}
k+j \\
j-1
\end{array}\right)\right),
\end{aligned}
$$

and the conclusion follows after the observation that $(k+1)\left(\begin{array}{c}k+j \\ j-1\end{array}\right)=j\left(\begin{array}{c}k+j \\ j\end{array}\right)$. 
Proof of Theorem 3. Rewrite Lemma 3 in the form

$$
N_{n, k}=\sum_{i=0}^{n-k}\left(\begin{array}{c}
n-i \\
k
\end{array}\right)(-1)^{n-k-i} h_{i} e_{n-i}
$$

and apply the homomorphism $\mathfrak{Z}$, using equation (6) and

$$
\mathfrak{Z}\left(h_{i}\right)=\frac{2\left(2^{2 i-1}-1\right)(-1)^{i-1} B_{2 i} \pi^{2 i}}{(2 i) !} .
$$

\section{Proof of Theorems 1 and 4}

From the introduction we recall the statement of Theorem 1:

$$
\begin{aligned}
E(2 n, k)=\frac{1}{2^{2(k-1)}} & \left(\begin{array}{c}
2 k-1 \\
k
\end{array}\right) \zeta(2 n) \\
& -\sum_{j=1}^{\left\lfloor\frac{k-1}{2}\right\rfloor} \frac{1}{2^{2 k-3}(2 j+1) B_{2 j}}\left(\begin{array}{c}
2 k-2 j-1 \\
k
\end{array}\right) \zeta(2 j) \zeta(2 n-2 j) .
\end{aligned}
$$

We note that Euler's formula (2) can be used to write the result in the alternative form

$$
E(2 n, k)=\sum_{j=0}^{\left\lfloor\frac{k-1}{2}\right\rfloor} \frac{(-1)^{j} \pi^{2 j} \zeta(2 n-2 j)}{2^{2 k-2 j-2}(2 j+1) !}\left(\begin{array}{c}
2 k-2 j-1 \\
k
\end{array}\right)
$$

which avoids mention of Bernoulli numbers.

We now expand out the generating function $F(t, s)$. We have

$$
\begin{aligned}
F(t, s)=\frac{1}{\sqrt{1-s} \sin \pi \sqrt{t}} & \sin (\pi \sqrt{t} \sqrt{1-s}) \\
= & \frac{\pi \sqrt{t}}{\sin \pi \sqrt{t}} \sum_{j=0}^{\infty} \frac{(-1)^{j} \pi^{2 j} t^{j}(1-s)^{j}}{(2 j+1) !}=\sum_{k=0}^{\infty} s^{k} G_{k}(t),
\end{aligned}
$$

where

$$
G_{k}(t)=(-1)^{k} \frac{\pi \sqrt{t}}{\sin \pi \sqrt{t}} \sum_{j \geq k} \frac{(-1)^{j} \pi^{2 j} t^{j}}{(2 j+1) !}\left(\begin{array}{l}
j \\
k
\end{array}\right) .
$$


Then Theorem 1 is equivalent to the statement that

$$
G_{k}(t)=\sum_{n \geq k} t^{n} \sum_{j=0}^{\left\lfloor\frac{k-1}{2}\right\rfloor} \frac{(-1)^{j} \pi^{2 j} \zeta(2 n-2 j)}{2^{2 k-2 j-2}(2 j+1) !}\left(\begin{array}{c}
2 k-2 j-1 \\
k
\end{array}\right)
$$

for all $k$. We can write the latter sum as

$$
\begin{gathered}
\sum_{j=0}^{\left\lfloor\frac{k-1}{2}\right\rfloor} \frac{\left(-4 \pi^{2} t\right)^{j}}{2^{2 k-2}(2 j+1) !}\left(\begin{array}{c}
2 k-2 j-1 \\
k
\end{array}\right) \sum_{n \geq j+1} \zeta(2 n-2 j) t^{n-j}- \\
\sum_{j=0}^{\left\lfloor\frac{k-1}{2}\right\rfloor} \frac{\left(-4 \pi^{2} t\right)^{j}}{2^{2 k-2}(2 j+1) !}\left(\begin{array}{c}
2 k-2 j-1 \\
k
\end{array}\right) \sum_{n=j+1}^{k-1} \zeta(2 n-2 j) t^{n-j}= \\
\frac{1}{2}(1-\pi \sqrt{t} \cot \pi \sqrt{t}) \sum_{j=0}^{\left\lfloor\frac{k-1}{2}\right\rfloor} \frac{\left(-4 \pi^{2} t\right)^{j}}{2^{2 k-2}(2 j+1) !}\left(\begin{array}{c}
2 k-2 j-1 \\
k
\end{array}\right)- \\
\sum_{j=0}^{\left\lfloor\frac{k-1}{2}\right\rfloor} \frac{\left(-4 \pi^{2} t\right)^{j}}{2^{2 k-2}(2 j+1) !}\left(\begin{array}{c}
2 k-2 j-1 \\
k
\end{array}\right) \sum_{n=j+1}^{k-1} \zeta(2 n-2 j) t^{n-j},
\end{gathered}
$$

where we have used the generating function

$$
\frac{1}{2}(1-\pi \sqrt{t} \cot \pi \sqrt{t})=\sum_{i=1}^{\infty} \zeta(2 i) t^{i} .
$$

Note that the last sum in (10) is a polynomial that cancels exactly those terms in

$$
\frac{1}{2}(1-\pi \sqrt{t} \cot \pi \sqrt{t}) \sum_{j=0}^{\left\lfloor\frac{k-1}{2}\right\rfloor} \frac{\left(-4 \pi^{2} t\right)^{j}}{2^{2 k-2}(2 j+1) !}\left(\begin{array}{c}
2 k-2 j-1 \\
k
\end{array}\right)
$$

of degree less than $k$. Thus, to prove Theorem 1 it suffices to show that

$$
G_{k}(t)=\text { terms of degree } \geq k \text { in expression (11). }
$$

From equation (9) it is evident that

$$
G_{k}(t)=\frac{\pi \sqrt{t}}{\sin \pi \sqrt{t}} \cdot \frac{(-t)^{k}}{k !} \cdot \frac{d^{k}}{d t^{k}}\left(\frac{\sin \pi \sqrt{t}}{\pi \sqrt{t}}\right) .
$$

We use this to obtain an explicit formula for $G_{k}(t)$. 
Lemma 4. For $k \geq 0$,

$$
G_{k}(t)=P_{k}\left(\pi^{2} t\right) \pi \sqrt{t} \cot \pi \sqrt{t}+Q_{k}\left(\pi^{2} t\right),
$$

where $P_{k}, Q_{k}$ are polynomials defined by

$$
\begin{aligned}
P_{k}(x) & =-\sum_{j=0}^{\left\lfloor\frac{k-1}{2}\right\rfloor} \frac{(-4 x)^{j}}{2^{2 k-1}(2 j+1) !}\left(\begin{array}{c}
2 k-2 j-1 \\
k
\end{array}\right) \\
Q_{k}(x) & =\sum_{j=0}^{\left\lfloor\frac{k}{2}\right\rfloor} \frac{(-4 x)^{j}}{2^{2 k}(2 j) !}\left(\begin{array}{c}
2 k-2 j \\
k
\end{array}\right) .
\end{aligned}
$$

Proof. In view of equation (12), the conclusion is equivalent to

$$
f^{(k)}(t)=(-1)^{k} k ! t^{-k} P_{k}\left(\pi^{2} t\right) \cos \pi \sqrt{t}+(-1)^{k} k ! t^{-k} Q_{k}\left(\pi^{2} t\right) f(t),
$$

where $f(t)=\sin \pi \sqrt{t} / \pi \sqrt{t}$. Differentiating, one sees that the polynomials $P_{k}$ and $Q_{k}$ are determined by the recurrence

$$
\begin{aligned}
& (k+1) P_{k+1}(x)=k P_{k}(x)-x P_{k}^{\prime}(x)-\frac{1}{2} Q_{k}(x) \\
& (k+1) Q_{k+1}(x)=\frac{2 k+1}{2} Q_{k}(x)-x Q_{k}^{\prime}(x)+\frac{x}{2} P_{k}(x)
\end{aligned}
$$

together with the initial conditions $P_{0}(x)=0, Q_{0}(x)=1$. The recurrence and initial conditions are satisfied by the explicit formulas above.

Proof of Theorem 1. Using Lemma 4, we have

$$
\begin{aligned}
G_{k}(t)= & -\sum_{j=0}^{\left\lfloor\frac{k-1}{2}\right\rfloor} \frac{\left(-4 \pi^{2} t\right)^{j}}{2^{2 k-1}(2 j+1) !}\left(\begin{array}{c}
2 k-2 j-1 \\
k
\end{array}\right) \pi \sqrt{t} \cot \pi \sqrt{t} \\
+\sum_{j=0}^{\left\lfloor\frac{k}{2}\right\rfloor} \frac{\left(-4 \pi^{2} t\right)^{j}}{2^{2 k}(2 j) !}\left(\begin{array}{c}
2 k-2 j \\
k
\end{array}\right)= & \\
& \begin{array}{c}
\frac{1}{2}(1-\pi \sqrt{t} \cot \pi \sqrt{t}) \sum_{j=0}^{\left\lfloor\frac{k-1}{2}\right\rfloor} \frac{\left(-4 \pi^{2} t\right)^{j}}{2^{2 k-2}(2 j+1) !}\left(\begin{array}{c}
2 k-2 j-1 \\
k
\end{array}\right) \\
+ \text { terms of degree }<k,
\end{array}
\end{aligned}
$$

and this completes the proof. 
Proof of Theorem 3. Using Theorem 1 in the form of equation (8), eliminate $\zeta(2 n-2 j)$ using Euler's formula (2) and then compare with Theorem 3 to get

$$
\begin{aligned}
\sum_{j=0}^{\left\lfloor\frac{k-1}{2}\right\rfloor} \frac{(-1)^{n-1} \pi^{2 n} B_{2 n-2 j}}{2^{2 k-2 n-1}(2 n-2 j) !(2 j+1) !}\left(\begin{array}{c}
2 k-2 j-1 \\
k
\end{array}\right)= \\
\frac{(-1)^{n-k-1} \pi^{2 n}}{(2 n+1) !} \sum_{i=0}^{n-k}\left(\begin{array}{c}
n-i \\
k
\end{array}\right)\left(\begin{array}{c}
2 n+1 \\
2 i
\end{array}\right) 2\left(2^{2 i-1}-1\right) B_{2 i}
\end{aligned}
$$

Now multiply both sides by $(-1)^{n-1} 2^{2 k-2 n-1} \pi^{-2 n}(2 n+1)$ ! and rewrite the factorials on the left-hand side as a binomial coefficient.

\section{References}

[1] T. Aoki, Y. Kombu, and Y. Ohno, A generating function for sums of multiple zeta values and its applications, Proc. Amer. Math. Soc. 136 (2008), 387-395.

[2] W. Y. C. Chen and L. H. Sun, Extended Zeilberger's algorithm for identities on Bernoulli and Euler polynomials, J. Number Theory 129 (2009), 2111-2132.

[3] L. Euler, Meditationes circa singulare serierum genus, Novi Comm. Acad. Sci. Petropol. 20 (1776), 140-186; reprinted in Opera Omnia, ser. I, vol. 15, B. G. Teubner, Berlin, 1927, pp. 217-267.

[4] H. Gangl, M. Kaneko, and D. Zagier, Double zeta values and modular forms, in Automorphic Forms and Zeta Functions, S. Böcherer et. al. (eds.), World Scientific, Singapore, 2006, pp. 71-106.

[5] M. E. Hoffman, Multiple harmonic series, Pacific J. Math. 152 (1992), 275-290.

[6] M. E. Hoffman, The algebra of multiple harmonic series, J. Algebra 194 (1997), 477-495.

[7] M. E. Hoffman, A character on the quasi-symmetric functions coming from multiple zeta values, Electron. J. Combin. 15 (2008), res. art. 97. 
[8] Y. Komori, K. Matsumoto and H. Tsumura, A study on multiple zeta values from the viewpoint of zeta-functions of root systems, Funct. Approx. Comment. Math. 51 (2014), 43-76.

[9] I. G. Macdonald, Symmetric Functions and Hall Polynomials, 2nd ed., Oxford Univ. Press, New York, 1995.

[10] T. Machide, Extended double shuffle relations and the generating function of triple zeta values of any fixed weight, Kyushu Math. J. 67 (2013), 281-307.

[11] Z. Shen and T. Cai, Some formulas for multiple zeta values, J. Number Theory 132 (2012), 314-323. 